Canadian Oncology

Nursing Journal

Revue canadienne

de soins infirmiers

en oncologie

Volume 28, Issue 3 • Summer 2018

elSSN: 2368-8076 


\section{Encourager les autosoins chez les patientes souffrant de réactions cutanées dues à la radiothérapie}

par Crystele Montpetit et Savitri Singh-Carlson

\section{RÉSUMÉ}

Contexte : Les patientes qui reçoivent de la radiothérapie contre un cancer du sein peuvent souffrir d'une desquamation humide et douloureuse de la peau située sous l'aisselle et dans le pli inframammaire. L'intensité des réactions cutanées varie d'une personne à l'autre et dépend de la dose totale de radiation, de la zone de traitement, du fractionnement de la dose quotidienne, de l'énergie du rayonnement émis et du type de radiothérapie, ainsi que d'autres facteurs individuels (Gosselin, 2010). Ces réactions dues à la radiothérapie rendent le port de vêtements et de sous-vêtements inconfortable et nuisent aux activités de tous les jours. Le pansement textile InterDry Ag est constitué d'un complexe antimicrobien qui évacue l'humidité et réduit le frottement, les démangeaisons et la sensation de brûlure. Bien que les propriétés de ce pansement soient hautement bénéfiques, il n'existe, à l'heure actuelle, aucune donnée probante montrant son utilité pour atténuer les réactions cutanées causées par la radiothérapie.

But : Ce projet évaluait l'intérêt potentiel du pansement InterDry Ag pour soulager linconfort dî aux réactions cutanées dont souffrent les patientes atteintes de cancer du sein sous radiothérapie.

Méthodologie : Un devis descriptif a été utilisé pour mieux comprendre l'efficacité du pansement InterDry Ag sur la réduction des effets secondaires causés par la radiothérapie mammaire. En tout, 20 femmes admissibles ont consenti à participer à l'étude. Tous les cinq jours, les effets du pansement étaient évalués par une infirmière en radio-oncologie à l'aide d'un questionnaire d'évaluation des symptômes cutanés, questionnaire mis au point par une équipe d'infirmières à partir d'observations cliniques.

Résultats : Une méthode personnalisée de soins de la peau a été instaurée à partir de la rétroaction de chaque patiente. Une relation de confiance s'est installée entre l'infirmière et la patiente, relation qui

\section{AU SUJET DES AUTEURES}

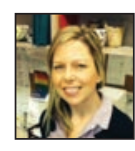

Auteure-ressource : Crystele Montpetit, inf. aut., B.Sc.inf., Infirmière en radiothérapie et oncologie systémique, British Columbia Cancer Agency, Fraser Valley Cancer Centre

Courriel : crystele.montpetit@bccancer.bc.ca Téléphone : 604-930-2098

Savitri Singh-Carlson, Ph.D., APHN-BC, FAAN, Vice-doyenne, Faculty Affairs and Diversity, University of Texas Health Sciences Center, San Antonio, School of Nursing, San Antonio, USA, 78248

Téléphone : 210-567-5883

Courriel : singhcarlson@uthscsa.edu a nourri d'autant plus le soutien apporté par les soins infirmiers. Certaines femmes se sont alors mises à parler aux infirmières $d u$ stress qu'elles vivaient, de même que de la douleur physique causée par ces réactions cutanées. Grâce aux observations des femmes et à l'adoption d'une approche axée sur les patientes, les infirmières ont pu leur offrir des ressources supplémentaires et encourager les pratiques d'autosoins.

Conflit d'intérêts : Les auteurs déclarent n'avoir aucun conflit d'intérêts lié à la rédaction du présent article.

\section{INTRODUCTION}

Clon les dernières statistiques, le type de cancer le plus fré- quent chez les Canadiennes est le cancer du sein. En effet, on estime que 25700 femmes ont reçu ce diagnostic en 2016 (Société canadienne du cancer, 2016). La recherche montre que, après les traitements contre ce cancer, la plupart des femmes présentent des effets secondaires psychologiques, physiques ou émotionnels négatifs qui éprouvent leur santé et leur bien-être (Budischewski, 2008; Smith, Singh-Carlson, Downie, Payeur et Wai, 2011; Tanay, 2013). L'inconfort qu'elles ressentent pendant la radiothérapie affecte leur qualité de vie et leurs activités quotidiennes.

La radiodermite est l'effet secondaire le plus fréquemment provoqué par la radiothérapie, effet qui peut entraîner des complications douloureuses chez les patientes atteintes d'un cancer du sein (Tanay, 2013). La radiothérapie et les réactions inflammatoires subséquentes sont des réactions physiopathologiques qui surviennent après l'irradiation de la peau, généralement au point d'entrée et de sortie du faisceau (BCCA, Symptom Management Guidelines, 2013). Les patientes sous radiothérapie mammaire peuvent également souffrir de réactions cutanées évolutives qui entraînent l'apparition d'une desquamation humide et douloureuse de l'aisselle et du pli inframammaire. Ces réactions rendent le port de vêtements et de sous-vêtements inconfortable pour les femmes et entravent leurs activités quotidiennes. Les symptômes de radiodermite varient de légers à intenses; ils sont évalués selon la version 4.03 de l'échelle «Common Terminology Criteria for Adverse Events » (critères communs de terminologie pour les réactions indésirables) du National Cancer Institute (NCI). En outre, si ces plaies ouvertes douloureuses ne sont pas traitées, elles peuvent entraîner des complications comme des infections bactériennes ou fongiques secondaires (KennedyEvans, Barbara et al., 2007). Ces patientes vivent également de la détresse émotionnelle à divers degrés, ainsi qu'un inconfort physique non négligeable (Schnur, Ouellette, Dilorenzo, Green et Montgomery, 2011). Des sensations de douleur, de brûlure, 
de démangeaison, de piqûre, de picotement et de lourdeur ont été rapportées par certaines de ces patientes (Paterson et al., 2012). L'intensité des symptômes peut s'aggraver jusqu'à deux semaines après la dernière séance de radiothérapie.

Le traitement des réactions cutanées causées par la radiothérapie dépend du niveau de toxicité et s'accompagne d'objectifs de gestion des symptômes et de prévention des infections. À la British Columbia Cancer Agency (BCCA), les infirmières en radio-oncologie ont fréquemment recours aux lignes directrices sur la gestion des symptômes (BCCA, Symptom Management Guidelines, 2013). Habituellement, on traite les réactions cutanées modérées à intenses par l'application de compresses de solution saline suivies d'une crème ou d'un hydratant délivré sur ordonnance, comme Flamazine ou de l'hydrocortisone. Cependant, pour plusieurs patientes, les infirmières en radio-oncologie de notre projet clinique ont observé que l'application d'une crème délivrée sur ordonnance semble difficile et salissante, d'autant plus qu'elle n'apporte pas toujours le soulagement espéré.

En général, les réactions dues à la radiothérapie se manifestent une à quatre semaines après le début du traitement et peuvent persister plusieurs semaines une fois qu'il est terminé (BCCA Symptom Management Guidelines: Radiation Dermatitis, 2013). De l'avis des spécialistes, les pansements imprégnés d'argent $(\mathrm{Ag})$ chassent l'humidité excessive des plis cutanés afin que la peau reste bien au sec tout en offrant une protection antimicrobienne efficace pendant cinq jours (Sibbald, 2013). Le doux tissu du pansement sert de barrière pour réduire le frottement, ce qui diminue les risques de déchirures cutanées. De plus, il permet de traiter les signes et symptômes de l'intertrigo, comme la macération, la dénudation, l'inflammation, le prurit, l'érythème et les lésions satellites afin d'éviter d'autres complications (Sibbald, 2013).

Il existe peu d'études sur l'utilisation clinique du pansement InterDry Ag dans la gestion des réactions cutanées dues à la radiothérapie, et ce, malgré ses propriétés médicinales potentiellement très bénéfiques pour ce groupe de patientes (Kumar, Juresic, Barton et Shafiq 2010). Pourtant, le coût des soins associés à l'utilisation du pansement InterDry Ag est environ deux fois moindre que celui des traitements sur ordonnance. Actuellement, ces réactions sont vues comme un aspect inévitable du traitement; par conséquent, en clinique de suivi, l'intervention infirmière vise principalement à soulager les symptômes (Porock, 1999). Selon d'autres rapports, différents produits (corticostéroïdes, sucralfate, pansements hydrocolloïdes et pansements perméables à la vapeur d'eau) ont fait l'objet de cinq études sur la gestion des symptômes (Paterson, 2012) qui ont conclu qu'aucun agent ne réduisait de façon significative les symptômes de réactions cutanées aiguës causées par la radiothérapie.

Pour combler la rareté des travaux de recherche sur l'efficacité du pansement InterDry Ag et sa capacité à évacuer l'humidité, un projet d'amélioration de la qualité a examiné son effet sur la guérison des réactions cutanées dues à la radiothérapie mammaire et sur le soulagement de l'inconfort lié aux symptômes. Le projet visait à évaluer la perception des patientes atteintes d'un cancer du sein par rapport à l'efficacité du pansement InterDry Ag dans le soulagement de l'inconfort causé par les réactions cutanées dues à la radiothérapie. Les conclusions du projet se sont révélées précieuses pour améliorer la qualité des soins prodigués à ce groupe de patientes souffrant de réactions cutanées dues à la radiothérapie et des complications qui y sont associées.

\section{SUIVI EN CLINIQUE DE SOINS INFIRMIERS}

La clinique de soins infirmiers du Fraser Valley Center (FVC) de la BCCA traite les symptômes secondaires de la radio et chimiothérapie (réactions cutanées, détresse causée par la douleur, nausées et autres dysfonctionnements physiopathologiques). Au début du traitement, les patientes reçoivent des feuillets d'information expliquant les effets secondaires de la radiothérapie. On leur conseille aussi d'appeler les infirmières de la clinique au besoin. Ces patientes peuvent être évaluées par une infirmière en radio-oncologie ou par leur radio-oncologue dans l'une des quatre cliniques externes ou à la clinique de suivi du service de radiothérapie.

Dans cette clinique, la pratique actuelle consiste à fixer un rendez-vous à la patiente avec son radio-oncologue quatre à huit semaines après le dernier traitement. L'évaluation infirmière des symptômes cutanés est essentielle pour mieux comprendre ce que vit chaque patiente et lui prodiguer les soins appropriés, notamment en encourageant les autosoins. Pour optimiser les résultats du présent projet, l'évaluation infirmière avait lieu au moins deux semaines après le dernier traitement de radiothérapie. À l'heure actuelle, l'infirmière en radio-oncologie de la clinique ne fait normalement pas d'évaluation de suivi; cela se produit seulement si nécessaire.

À la fin du traitement, les radiothérapeutes peuvent aiguiller les patientes vers une infirmière en radio-oncologie, qui évaluera les réactions cutanées individuelles. L'évaluation est généralement effectuée au service de suivi de la clinique de radiothérapie. L'enseignement aux patients est un aspect important de cette rencontre, car c'est à ce moment que les patientes reçoivent de l'information, verbale ou écrite, sur la gestion des effets secondaires associés aux réactions cutanées. À cette occasion, l'infirmière évalue également toute éventuelle détresse émotionnelle ou malnutrition qui pourraient nuire au processus de guérison d'une plaie. Pendant l'évaluation, l'infirmière en radio-oncologie informe les patientes sur les soins de la peau irradiée et l'application quotidienne d'une compresse de solution saline. Les directives post-traitement doivent être écrites et claires, et l'infirmière doit vérifier que la patiente comprend bien les autosoins à se prodiguer en cas de desquamation humide (Laffin et al., 2015).

\section{MÉTHODOLOGIE}

Un devis descriptif a été adopté pour mieux comprendre l'expérience des patientes atteintes d'un cancer du sein qui utilisaient le pansement InterDry Ag pour soulager les symptômes cutanés dus à la radiothérapie active. La méthode dite « d'évaluation par boucle de rétroaction » convenait bien au projet, car elle permettait de mettre en pratique les données obtenues pour améliorer la qualité des soins (Gotz et SinghCarlson, 2014). 
À partir des observations cliniques de patientes atteintes de cancer du sein présentant une radiodermite complexe formulées par l'équipe de soins infirmiers, un questionnaire à questions ouvertes a été élaboré pour la collecte de données. Le questionnaire comprenait à la fois des questions structurées et des questions ouvertes afin de recueillir les réponses qualitatives des participantes décrivant leur expérience avec le pansement (voir les annexes A et B). Le questionnaire (voir l'annexe C) proposait des réponses graduées selon une échelle de Likert pour mesurer l'efficacité possible du pansement InterDry Ag à améliorer le confort des patientes.

Après obtention des approbations éthiques nécessaires, les patientes ont été recrutées selon leur admissibilité et leur volonté à participer à l'étude. Les critères d'admissibilité étaient les suivants : a) recevoir un traitement de radiothérapie mammaire au FVC; b) n'avoir aucune allergie connue à l'argent; c) souffrir de réactions cutanées dues à la radiothérapie; d) parler et lire l'anglais couramment. Une infirmière en radio-oncologie distribuait une lettre présentant le projet d'évaluation pour recruter les candidates potentielles au début de leur traitement de radiothérapie. Après avoir obtenu leur consentement écrit, elle leur remettait les instructions et évaluait hebdomadairement les réactions cutanées. Le pansement InterDry Ag n'était recommandé que pour les réactions cutanées s'accompagnant d'un érythème, d'un prurit ou d'une sensation de brûlure jugés modérés par la patiente. Le pansement était utilisé durant deux semaines après la fin du traitement ou l'apparition d'une desquamation humide.

\section{Déroulement de l'étude}

Chaque semaine, pendant le traitement actif, toutes les participantes présentant un érythème mammaire étaient évaluées et recevaient de l'information sur les compresses de solution saline, les hydratants et l'application du pansement InterDry Ag.

Le pansement InterDry Ag était recommandé en cas d'inconfort et d'érythème modéré pour soulager les sensations de brûlure, de démangeaison et de malaise général. S'il n'y avait pas de plaie ouverte, le pansement était apposé sur toute la surface du sein. On conseillait également aux patientes de continuer l'application régulière et périodique de compresses de solution saline et d'une crème hydratante. Avant l'application du pansement, la crème devait être totalement absorbée et la peau bien asséchée.

L'évaluation standard hebdomadaire vérifiait l'état de la peau et l'utilisation du pansement InterDry Ag à l'aide de l'outil Common Terminology Criteria for Adverse Events du NCI (version 4.03) ainsi que d'un questionnaire administré aux participantes par une infirmière en radio-oncologie (annexe A). À cette étape, le confort des participantes était également sondé. $\mathrm{Si}$, à un moment du traitement, la patiente souffrait de desquamation humide, le radio-oncologue lui prescrivait une pommade de Flamazine qu'elle devait appliquer sur toutes les plaies ouvertes; le pansement InterDry Ag n'était toutefois pas apposé sur les plaies ouvertes. Souvent, le traitement standard du prurit se résumait à la prescription d'une crème d'hydrocortisone, il revenait alors au radio-oncologue de juger de la situation. Dans notre étude, trois patientes appliquaient de l'hydrocortisone avant d'apposer le pansement InterDry Ag.

L'évaluation dermatologique vérifiait l'intégrité des trois principales zones touchées par la radiothérapie mammaire : l'aisselle, le pli inframammaire et la paroi thoracique. L'érythème, la douleur et le prurit étaient évalués et cotés « absents, légers, modérés ou intenses ». La collecte d'information était orientée selon les observations cliniques de l'infirmière en radio-oncologie et la rétroaction des patientes. Chaque participante a subi de deux à cinq évaluations dermatologiques. Le nombre de séances dépendait du moment où la patiente avait commencé à utiliser le pansement InterDry Ag et du nombre total de traitements de radiothérapie reçus. Pendant ces rencontres, les participantes pouvaient discuter de leurs inquiétudes. Pour s'adapter aux besoins individuels des participantes, chaque rendez-vous durait entre quinze et trente minutes.

Le pansement InterDry Ag était remplacé tous les cinq jours lors des rendez-vous d'évaluation. Au dernier traitement de radiothérapie, les participantes recevaient deux pansements InterDry Ag supplémentaires, ainsi que le matériel nécessaire pour le maintenir en place. Elles pouvaient ainsi panser leurs plaies en attendant la prochaine visite de suivi.

L'infirmière en radio-oncologie de la clinique de suivi téléphonait aux participantes une semaine après leur dernier traitement de radiothérapie pour recueillir de l'information sur leurs expériences à l'aide d'un questionnaire standardisé (annexe B) de onze questions portant sur l'efficacité du pansement InterDry Ag pour soulager l'inconfort. Les participantes avaient alors l'occasion de poser leurs questions ou d'exprimer leurs inquiétudes au sujet du pansement et de l'état de leur peau. Deux semaines après la radiothérapie, l'infirmière en radio-oncologie procédait à une évaluation dermatologique et administrait un questionnaire post-traitement (annexe C). À cette occasion, les participantes pouvaient formuler tout autre commentaire ou suggestion supplémentaire inspiré de leur expérience avec le pansement InterDry Ag.

\section{Analyse des données}

Les réponses au questionnaire ont été analysées à l'aide de statistiques descriptives. L'infirmière en radio-oncologie a recueilli des données sur l'érythème, la douleur et le prurit dans la région de l'aisselle (AI), du pli inframammaire (PI) et de la paroi thoracique (PT). Les données ont ensuite été saisies dans Excel 2007, et chaque effet secondaire et point à évaluer, coté absent $=0$, léger $=1$, modéré $=2$ ou intense $=3$. Les réponses des patientes (annexe C) se voyaient attribuer une valeur numérique située entre un (1) et six (6) selon une échelle de Likert : fortement en accord $=1$; en accord $=2$; indécise $=3$; en désaccord $=4$; fortement en désaccord $=5$; ne s'applique pas $=6$. Chaque réponse comptait pour un point. L'auteure (Crystele Montpetit) a vérifié et saisi les données du questionnaire. Une seconde personne a ensuite vérifié l'exactitude des données saisies avant leur analyse statistique dans Excel 2007. Les réponses qualitatives des participantes ont été résumées, et des citations, extraites, pour appuyer les données. L'analyse du contenu a permis de catégoriser les réponses qualitatives des participantes (Stemler, 2017). 


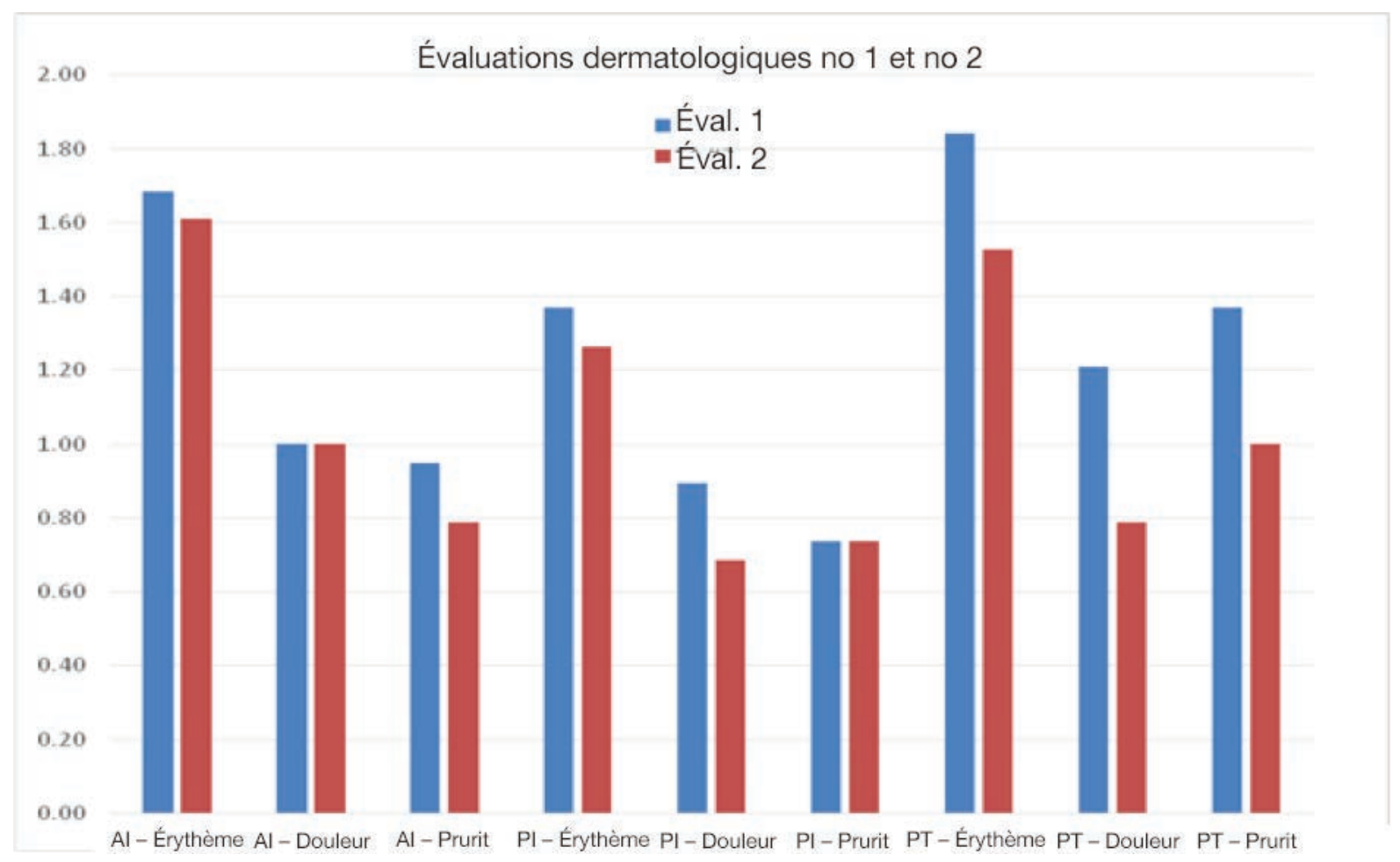

Figure 1 : Évaluation dermatologique initiale (temps 1) et subséquente (temps 2) ( $\mathrm{N}=18$ )

\section{RÉSULTATS}

Au départ, vingt patientes admissibles ont accepté de participer au projet d'évaluation; toutefois, l'une d'entre elles s'est désistée pour des motifs personnels et une autre ne s'est pas présentée à son rendez-vous de suivi. Par conséquent, dix-huit participantes ont complété la première et la seconde évaluation dermatologique, ainsi que l'entrevue téléphonique et le questionnaire de suivi administré deux semaines après la fin du traitement de radiothérapie.

\section{Données démographiques}

Lâge médian des participantes était de 42 ans (et variait entre 36 et 74 ans). Dix-sept participantes (91\%) étaient non-fumeuses. La taille des seins était très variable (entre le bonnet $\mathrm{A}$ et le bonnet $\mathrm{E}$, environ); certaines avaient le teint foncé, d'autres, le teint pâle. L'étude n'excluait pas celles qui recevaient de la chimiothérapie en plus de la radiothérapie. Neuf participantes $(15 \%)$ avaient une poitrine volumineuse nécessitant un dispositif de soutien supplémentaire, comme un moule ou une coquille, pour stabiliser le sein pendant la radiothérapie. En outre, $20 \%(\mathrm{~N}=3)$ des participantes avaient passé la première étape d'une reconstruction mammaire avant les traitements de radiothérapie. Les plans de traitement différaient d'une femme à l'autre, notamment au sujet du nombre de traitements (entre 15 et 25), de même que la dose par fraction. Le nombre médian de fractions était de vingt-cinq pour une irradiation totale de 5000 cGy; la dose médiane par fraction était de 200 cGY.

\section{Résultats de l'évaluation}

La figure 1 compare les deux premières évaluations dermatologiques réalisées par l'infirmière en radio-oncologie.
La première a eu lieu avant l'utilisation du pansement InterDry Ag et la seconde, cinq jours après l'application du pansement. Les participantes ont rapporté une diminution de la douleur ressentie à la paroi thoracique et au pli inframammaire après l'installation du pansement. Certaines participantes ont même remarqué une réduction de l'inconfort le jour même de l'application, ainsi qu'un soulagement du prurit au thorax et à l'aisselle.

À chaque évaluation dermatologique, les participantes ont répondu à une série de io questions (annexe $\mathrm{A}$ ). Cinq jours après le début de l'utilisation d'InterDry Ag, on a demandé aux patientes si le pansement soulageait leur inconfort. Dix-huit $(94,7 \%)$ d'entre elle ont répondu « oui » et $84,2 \%(\mathrm{~N}=16)$ ont affirmé que l'état de leur peau s'était amélioré depuis qu'elles utilisaient le pansement. Lorsqu'on a demandé aux participantes si le pansement apaisait la douleur, $63,2 \%(\mathrm{~N}=12)$ ont répondu « oui » et $36,8 \%(N=7)$ étaient indécises. Plusieurs $(26,3 \%)(\mathrm{N}=5)$ ont dit avoir de la difficulté à maintenir le pansement en place, que ce soit à l'aide de la technique préconisée ou d'une méthode qu'elles avaient elles-mêmes improvisée. En revanche, $63,2 \%(\mathrm{~N}=11)$ n'ont eu aucun problème et 10,5\% $(\mathrm{N}=2)$ n'ont éprouvé des difficultés qu'à l'occasion.

L'infirmière en radio-oncologie téléphonait à chaque participante pour un suivi une semaine après le dernier traitement de radiothérapie (annexe B); l'infirmière posait alors une série de 10 questions (voir le tableau 1). Dix-huit participantes ont répondu à ce questionnaire téléphonique. Parmi celles-ci, 94 \% $(\mathrm{N}=17)$ avaient eu recours au pansement InterDry Ag une semaine après le dernier traitement de radiothérapie en raison d'une desquamation humide ou sèche de la peau. Dix-sept participantes $(94 \%)$ ont trouvé le pansement facile à utiliser et 


\begin{tabular}{|c|c|c|c|c|c|}
\hline Questions & Oui & Non & Amélioration & Détérioration & $\begin{array}{l}\text { Aucun } \\
\text { changement }\end{array}$ \\
\hline $\begin{array}{l}\text { 1. Utilisez-vous le pansement InterDry } \\
\text { actuellement? (Si non, passez à la } \\
\text { question 10.) }\end{array}$ & $94,1 \%$ & $5,9 \%$ & $0,0 \%$ & $0,0 \%$ & $0,0 \%$ \\
\hline $\begin{array}{l}\text { 2. Trouvez-vous le pansement simple à } \\
\text { utiliser? }\end{array}$ & $93,8 \%$ & $6,3 \%$ & $0,0 \%$ & $0,0 \%$ & $0,0 \%$ \\
\hline $\begin{array}{l}\text { 3. La rougeur de votre peau s'est-elle } \\
\text { améliorée ou détériorée depuis votre } \\
\text { dernier traitement? }\end{array}$ & $0,0 \%$ & $0,0 \%$ & $43,8 \%$ & $43,8 \%$ & $12,5 \%$ \\
\hline $\begin{array}{l}\text { 4. Depuis le dernier traitement, avez-vous } \\
\text { des plaies ouvertes (plaques suintantes)? }\end{array}$ & $31,3 \%$ & $68,8 \%$ & $0,0 \%$ & $0,0 \%$ & $0,0 \%$ \\
\hline $\begin{array}{l}\text { 5. Avez-vous changé le pansement plus } \\
\text { souvent qu'aux cinq jours? }\end{array}$ & $12,5 \%$ & $87,5 \%$ & $0,0 \%$ & $0,0 \%$ & $0,0 \%$ \\
\hline 6. Ressentez-vous de la douleur? & $86,7 \%$ & $13,3 \%$ & $0,0 \%$ & $0,0 \%$ & $0,0 \%$ \\
\hline 7. Le pansement est-il confortable? & $100,0 \%$ & $0,0 \%$ & $0,0 \%$ & $0,0 \%$ & $0,0 \%$ \\
\hline $\begin{array}{l}\text { 8. Selon vous, le pansement a-t-il aidé la } \\
\text { peau à guérir? }\end{array}$ & $86,7 \%$ & $6,7 \%$ & $6,7 \%$ & $0,0 \%$ & $0,0 \%$ \\
\hline 9. Le pansement soulage-t-il votre peau? & $100,0 \%$ & $0,0 \%$ & $0,0 \%$ & $0,0 \%$ & $0,0 \%$ \\
\hline $\begin{array}{l}\text { 10. Pourquoi avez-vous cessé d'utiliser } \\
\text { le pansement? (Ne s'applique que si la } \\
\text { personne n'utilise pas le pansement.) }\end{array}$ & $0,0 \%$ & $0,0 \%$ & $0,0 \%$ & $0,0 \%$ & $0,0 \%$ \\
\hline $\begin{array}{l}\text { 11. Utilisez-vous d'autres pansements ou } \\
\text { crèmes? }\end{array}$ & $93,8 \%$ & $6,3 \%$ & $0,0 \%$ & $0,0 \%$ & $0,0 \%$ \\
\hline
\end{tabular}

toutes les participantes ont vu l'état de leur peau s'améliorer grâce au pansement. Seize participantes (88 \%) étaient d'avis que le pansement avait favorisé la guérison et toutes les participantes l'ont trouvé confortable.

Le tableau 2 précise les caractéristiques du pansement InterDry Ag, de même que ses effets sur les sensations de douleur, de démangeaison et de brûlure dues à la réaction cutanée. Le questionnaire a été rempli lors du rendez-vous de suivi avec l'infirmière à la clinique externe du FVC. Deux participantes seulement ont eu besoin de Flamazine pendant leur traitement : elles souffraient de réactions cutanées graves et d'une desquamation humide. La Flamazine et l'hydrocortisone sont des pommades médicamenteuses topiques habituellement prescrites par le radio-oncologue pour soulager l'inconfort intense et prévenir l'infection. Dans notre étude, elles étaient réservées à celles qui présentaient une dermatite aiguë et une desquamation humide. La détresse et la toxicité due à la radiothérapie ont affecté les participantes à divers degrés. Trois d'entre elles ont choisi d'appliquer de l'hydrocortisone en plus du pansement InterDry Ag. Toutefois, le prurit a persisté malgré la prescription d'hydrocortisone; les participantes craignaient donc que les démangeaisons empirent si elles cessaient d'utiliser la pommade. On leur a conseillé d'attendre que l'hydrocortisone soit complètement absorbée par la peau avant d'apposer le pansement InterDry Ag. Pour certaines participantes, le soulagement du prurit avec le pansement était franchement marqué. Les participantes s'inquiétaient d'une possible intensification du prurit si elles cessaient d'appliquer la pommade.

\section{Expériences personnelles des participantes}

$\mathrm{Au}$ début du traitement, une méthode de soins dermatologiques personnalisée a été établie avec chaque participante en préconisant notamment l'application de compresses de solution saline et d'un hydratant. On passait également en revue l'utilisation appropriée du pansement. Lors des visites de suivi, qui avaient lieu tous les cinq jours, les participantes pouvaient poser des questions et exprimer leurs préoccupations à l'infirmière en radio-oncologie. Aux dires des patientes, elles avaient moins d'anxiété et appréciaient encore plus leurs soins depuis que les contacts avec l'infirmière en radio-oncologie étaient plus fréquents. À leur avis, les visites de suivi diminuaient leur anxiété; cet aspect n'a toutefois pas pu être vérifié objectivement puisque la clinique n'utilise pas dans sa pratique d'échelle qui mesure l'anxiété. 
Tableau 2 : Résultats du sondage des patientes $(N=18)$

\begin{tabular}{|c|c|c|c|c|c|c|}
\hline $\begin{array}{l}\text { Dans quelle mesure êtes-vous d'accord avec } \\
\text { l'énoncé? }\end{array}$ & $\begin{array}{l}\text { Fortement } \\
\text { en accord }\end{array}$ & En accord & Indécise & En désaccord & $\begin{array}{l}\text { Fortement en } \\
\text { désaccord }\end{array}$ & Ne s'applique pas \\
\hline 1. InterDry Ag est facile à utiliser. & $39 \%$ & $61 \%$ & $0 \%$ & $0 \%$ & $0 \%$ & $0 \%$ \\
\hline 2. InterDry Ag soulage la douleur. & $50 \%$ & $40 \%$ & $0 \%$ & $5 \%$ & $0 \%$ & $5 \%$ \\
\hline 3. InterDry Ag soulage la démangeaison. & $50 \%$ & $33 \%$ & $6 \%$ & $11 \%$ & $0 \%$ & $0 \%$ \\
\hline 5. Le pansement soulage ma peau. & $67 \%$ & $28 \%$ & $5 \%$ & $0 \%$ & $0 \%$ & $0 \%$ \\
\hline $\begin{array}{l}\text { 6. Je crois que le pansement a aidé ma peau } \\
\text { à guérir. }\end{array}$ & $61 \%$ & $17 \%$ & $22 \%$ & $0 \%$ & $0 \%$ & $0 \%$ \\
\hline $\begin{array}{l}\text { 7. Le pansement a soulagé la sensation de } \\
\text { brûlure. }\end{array}$ & $56 \%$ & $22 \%$ & $11 \%$ & $0 \%$ & $0 \%$ & $11 \%$ \\
\hline $\begin{array}{l}\text { 9. Je suis plus confortable lorsque je porte le } \\
\text { pansement. }\end{array}$ & $72 \%$ & $17 \%$ & $11 \%$ & $0 \%$ & $0 \%$ & $0 \%$ \\
\hline $\begin{array}{l}\text { 10. Je recommande l'utilisation du } \\
\text { pansement. }\end{array}$ & $78 \%$ & $17 \%$ & $5 \%$ & $0 \%$ & $0 \%$ & $0 \%$ \\
\hline
\end{tabular}

De l'avis personnel des participantes atteintes de réaction cutanée, le pansement InterDry Ag présentait à la fois des avantages et des difficultés. La plupart des participantes ont aimé les soins reçus et les effets positifs du pansement. Certaines ont exprimé le besoin de porter le pansement pour prévenir les effets secondaires douloureux de la radiothérapie; toutefois, le pansement était plutôt difficile à maintenir en place. Les sous-vêtements ont donc dû être modifiés et le pansement remplacé tous les cinq jours. Le tableau 3 présente les avantages et les difficultés de l'utilisation du pansement avec des citations textuelles.

\section{DISCUSSION}

Le projet a été entrepris pour évaluer l'efficacité du pansement InterDry Ag sur les symptômes des patientes atteintes d'un cancer sous radiothérapie. Tanay (2013) rapporte que la radiothérapie entraîne, chez la plupart des femmes, des effets secondaires physiques causant une détresse émotionnelle qui se manifeste sous forme de tension, de gêne, d'isolement, de peur et d'incertitude. Manifestement, les patientes ont besoin de bâtir une relation de confiance avec leur professionnel de la santé dès la pose du diagnostic. Elles ont aussi besoin de maintenir cette relation pendant toute la durée du traitement afin de pouvoir solliciter des conseils médicaux et de recevoir du soutien émotionnel (Bonito, 2013). Ainsi, les participantes au projet ont pu raconter leurs expériences intimes, notamment les difficultés associées au diagnostic de cancer du sein et à la radiothérapie. La relation de confiance contribue à diminuer significativement le niveau de détresse, particulièrement au début des traitements de radiothérapie (Bonito, 2013). Selon
Banning (2013), le sentiment d'impuissance s'installe lorsque la relation entre le professionnel de la santé et le patient n'est pas égalitaire dès le diagnostic et l'amorce du traitement, du moins dans la perception qu'en a le grand public; pour être à l'aise, les patients doivent se sentir soutenus et en contrôle.

La radiothérapie entraîne souvent une perte d'intégrité de la peau, ce qui enclenche un processus de cicatrisation des plaies, un phénomène à la fois complexe et bien orchestré (Kramer, 2010). Une étude comparative randomisée (Laffin et al., 2015) confirme que jusqu'à 4I \% des patientes atteintes d'un cancer du sein vivant sous les tropiques risquent de développer une desquamation humide pendant le traitement de radiothérapie ou tout de suite après. Cette situation exige la mise en place de différentes stratégies de gestion du cancer, notamment le recours à divers pansements avec analgésiques. Selon les travaux de Patterson et collègues (2012), il est possible que les différences de perception face à la réaction cutanée s'expliquent davantage par l'origine ethnique que par des facteurs quantifiables tels que l'intensité ou l'emplacement de l'érythème ou de la desquamation.

Une analyse exhaustive a, quant à elle, révélé que les changements cutanés causés par la radiothérapie mammaire influent négativement sur plusieurs dimensions de la qualité de vie des femmes, notamment leur bien-être physique et fonctionnel, de même que sur leur niveau de satisfaction par rapport au traitement (Schnur, Ouellette, Dilorenzo, Green et Montgomery, 2011). Les participantes à notre projet d'évaluation se sentaient en sécurité, ce qui leur donnait l'impression de maîtriser les répercussions émotionnelles et psychosociales des plaies cutanées, conséquences du diagnostic et du traitement du cancer du sein. Les évaluations hebdomadaires 
Tableau 3 : Citations textuelles des participantes

\section{Avantages et difficultés tels que perçus par les patientes}

Avantages

- En utilisant le pansement, je n'avais pas besoin de prendre des antidouleurs. Lorsque je ne le porte pas, je suis très inconfortable. Le soulagement se produit en quelques minutes. Je ne sais pas ce que jaurais fait sans le pansement.

- Je suis satisfaite du pansement InterDry Ag et je le recommanderais à d'autres.

- En rentrant chez moi, j’ai remarqué que les symptômes de démangeaison et de brûlure ne me dérangeaient plus. Les désagréments avaient disparu.

- Un grand soulagement. Une sensation de fraîcheur et une diminution de l'irritation.

- Je ne peux pas imaginer l'intensité des brûlures de radiothérapie sans le pansement InterDry. Lorsque je l'enlevais, la douleur était insupportable. Dès que je le remettais, la douleur et la démangeaison disparaissaient instantanément.

- Le tissu m'a apporté tellement de confort pendant le processus de guérison.

- Au début, les brûlures me faisaient pleurer. Le pansement m'a rendu mon confort.

- Merci beaucoup de nous fournir ce pansement médical fantastique. J'ai vraiment apprécié l'excellent service. Encore une fois, merci pour votre temps et votre considération.

- Sans le pansement, je n'aurais pas été capable d'aller au bout du traitement.

- Au début, les brûlures me faisaient pleurer, mais grâce au pansement, j'ai retrouvé le confort. InterDry empêchait la formation de cloques et soulageait la sensation omniprésente de brûlure.

- Je suis tellement contente et chanceuse d'avoir participé à ce projet/cette étude. Le pansement m'a apporté tellement de confort pendant le processus de guérison. J'espère sincèrement qu'il pourra être offert à tous les patients en radiothérapie à l'avenir.

- Le jour oùj'ai reçu le pansement, ma peau était irritée, elle me démangeait et me brûlait. En rentrant chez moi, j’ai remarqué que les symptômes ne me gênaient plus.

Difficultés

- Impossible de garder le pansement en place sous le bras. Le pansement fonctionnait bien sur la poitrine et aux endroits où il restait en place.

- Un grand soulagement. Mais il faut trouver une façon de mieux le fixer.

effectuées par l'infirmière de la clinique apportaient un sentiment de contrôle et un soutien thérapeutique aux patientes qui devaient gérer les effets secondaires émotionnels et physiques de leurs traitements. Les participantes du projet ont rapporté de la satisfaction par rapport aux évaluations hebdomadaires, qui les aidaient à entretenir une bonne relation avec l'infirmière. Le questionnaire téléphonique semblait aussi jouer un rôle important pour établir le lien de confiance et encourager les patientes à prendre leurs soins en charge. Selon une étude récente menée par Bonito (2013), lorsque leurs professionnels de la santé abordent les répercussions du cancer, les patients voient leur qualité de vie améliorée et sont plus satisfaits de ces professionnels lorsque ces derniers abordent les répercussions du cancer. Cela leur donne l'espace pour raconter leurs expériences. En outre, pour Noble-Adams (1999), l'évaluation dermatologique holistique menée par l'infirmière optimise les soins de la peau pendant la période suivant la radiothérapie. Les participantes ont exprimé leur satisfaction envers les soins reçus et ont apprécié le fait de pouvoir parler ouvertement de leur bien-être émotionnel.

\section{LIMITES}

Le projet d'évaluation présente certaines limites, notamment la taille restreinte de l'échantillon et l'hétérogénéité du groupe de participantes. Si ce groupe avait été plus diversifié du point de vue de l'origine ethnique et de la langue, peut-être aurions-nous pu repérer des lacunes dans les soins offerts aux patientes souffrant de radiodermite. Peut-être les patientes d'autres origines ethniques auraient-elles pu nous orienter vers des pratiques différentes de gestion des autosoins. Les projets d'évaluation à venir devraient être réalisés auprès de différents groupes pour déterminer le type de matériel éducatif convenant aux femmes non anglophones. De plus, le projet aurait gagné à inclure les membres de la famille qui jouent le rôle de proches aidants. Il se prêterait également à une étude randomisée avec groupe témoin et outils de mesure validés. Les outils de collecte des données utilisés dans le cadre du projet ont été élaborés à partir d'observations cliniques, mais n’ont pas été validés. La collecte d'autres données démographiques permettrait d'obtenir des renseignements précieux sur la toxicité cutanée et l'utilisation du pansement InterDry Ag. Une étude à long terme aurait aussi mis au jour des éléments clés des pratiques d'autosoins et d'autoefficacité.

\section{IMPLICATIONS POUR LA PRATIQUE}

Le pansement InterDry Ag a prouvé qu'il réduisait l'inconfort ressenti par les femmes sous radiothérapie mammaire. Une étude de plus grande envergure, réalisée selon un devis clinique randomisé, pourrait mieux circonscrire les avantages du pansement InterDry Ag et sa capacité à préserver l'intégrité de la peau comparativement aux méthodes habituelles de traitement de la radiodermite. Dans notre petit groupe, la majorité des participantes n'ont pas eu besoin de Flamazine; le pansement InterDry Ag semble donc être rentable. Répondre aux besoins physiques et émotionnels des patientes pendant cette période difficile de leur vie est essentiel au maintien de leur bien-être. Dans cette optique, l'enseignement d'habiletés d'autosoins aiderait les patientes et leur apporterait un sentiment de contrôle. Le pansement InterDry Ag a permis à de nombreuses participantes de vaquer à leurs tâches quotidiennes sans ressentir la douleur et l'inconfort permanent des réactions cutanées dues à la radiothérapie.

\section{CONCLUSION}

L'utilisation du pansement InterDry Ag pour soulager les réactions cutanées du sein s'est révélée bénéfique pour le 
confort des patientes de notre petite cohorte. Il est extrêmement important, pour les patientes, de continuer à vaquer à leurs activités au quotidien après avoir reçu un diagnostic de cancer du sein et les traitements subséquents. Grâce à la relation de confiance établie avec l'infirmière en radio-oncologie, la plupart des participantes ont su exprimer leurs inquiétudes et leurs peurs, trouvant ainsi le réconfort nécessaire pour apaiser leur anxiété. Cette approche, en étant centrée sur les

\section{RÉFÉRENCES}

Banning, M.G. \& Gumley, V. (2013). Case studies of patient interactions, care provision and the impact of emotion: A qualitative study. Journal of Cancer Education, 28, 656-661.

Bonito, A.H., Horowitz, N., McCorkle, R., \& Chagpar, A.B. (2013). Do healthcare professionals discuss the emotional impact of cancer with patients? Psycho-Oncology, 22, 2046-2050.

British Columbia Cancer Agency (2013). British Columbia Symptom Management Guidelines. Retrieved from http://www.bccancer. bc.ca/health-professionals/clinical-resources/nursing/ symptom-management

Budischewski, K.F. (2008). Quality of life of breast cancer patients in the course of adjuvant radiotherapy. Support Care Cancer, 16, 299-304.

Canadian Cancer Statistics. (n.d.). Breast cancer statistics. Retrieved from http://www.cancer.ca/en/cancer-information/cancer-type/ breast/statistics/?region $=\mathrm{bc}$

Gosselin, T.K., Schneider, S.M., Plambeck, M.A., \& Rowe, K. (2010). A prospective randomized, placebo-controlled skin care study in women diagnosed with breast cancer undergoing radiation therapy. Oncol Nurs Forum, 37(5), 619-626.

Gotz, C., \& Singh-Carlson, S. (2014). Mixed methods approach to program evaluation: Measuring impact of a cancer support program. Journal of Women's Health, Issues a Care, 3(3), 1-4. http://dx.doi.org/10.4172/2325-9795.1000151

Kennedy-Evans, K.L., Viggiano, B., Henn, T., \& Smith, D. (2007). Multi-site feasibility study using a new textile with silver for management of skin conditions located in skin folds. Journal of Wound, Ostomy a Continence Nursing, 34(3S), S68.

Kramer, A., Hubner, N.O., Assadian, O., Mulder, G. (2010). Polihexanide-perspectives on clinical wound antisepsis. Skin Pharmacology and Physiology, 23(Suppl 1), 1-3.

Kumar, S., Juresic, E., Barton, M., \& Shafiq, J. (2010). Management of skin toxicity during radiation therapy: A review of the evidence. Journal of Medical Imaging and Radiation Oncology, 54(3), 264-79. patientes, a permis aux femmes atteintes d'un cancer de sein de recevoir des soins personnalisés pendant la phase de diagnostic et de traitement.

\section{CONFLITS D'INTÉRÊTT}

Les auteurs n'ont aucun conflit d'intérêt à déclarer lié aux produits utilisés pour ce projet.

Laffin, N.S., Smyth, W., Heyer, E., Fasugba, O., Abernethy, G., \& Gardner, A. (2015). Effectiveness and acceptability of a moisturizing cream and a barrier cream during radiation therapy for breast cancer in the tropics. Cancer Nursing, 38(3), 205-2014.

NCI CTCAE (version 4.03). Dermatitis radiation (2013). Retrieved from, $\mathrm{H}$ :|everyone $\mid$ nursing $\backslash$ references and guidelines $\backslash$ Symptom Management Guidelines\radiation dermatitis

Noble-Adams, R. (1999). Radiation-induced reactions. 1: An examination of the phenomenon. British Journal of Nursing, 8(17), 1134-40.

Porock, D., \& Kristjanson, L. (1999). Skin reactions during radiotherapy for breast cancer: The use and impact of topical agents and dressings, European Journal of Cancer Care, 8, 143-153.

Paterson, D., Poonam, P., Bennett, N.C, Peszynski, R., Beekhuizen, M.J.V., Jasperse, M.L., \& Herst, M. (2012). Randomized intrapatient controlled trial of Mepilex Lite Dressings versus Aqueous Cream in managing radiation-induced skin reactions postmastectomy. Cancer Science \& Therapy, 4(11), 347-356.

Schnur, J.O., Ouellette, S.C., Dilorenzo, T.A., Green, S., \& Montgomery, G.H. (2011). A qualitative analysis of acute skin toxicity among breast cancer radiotherapy patients. PsychoOncology, 20, 260-268.

Sibbald, G.R., Kelley, J., Kennedy-Evans, Labrecque, C., \& Waters, N. (2013). A practical approach to the prevention and management of intertrigo, or moisture-associated skin damage, due to perspiration: Expert consensus on best practice. Wound Care Canada, 11(2).

Smith, L.S., Singh-Carlson, S., Downie, L., Payeur, N., \& Wai, E. (2011). Survivors of breast cancer: Patient perspectives on survivorship care planning. Journal of Cancer Survivorship, 5, 337-44.

Stemler, S. (2001). An overview of content analysis. Practical Assess Res Evaluation, 7(17). Retrieved from http://pareonline.net/getvn. asp? $\mathrm{v}=7 \& \mathrm{n}=17$

Tanay, M.W., Wiseman, T., Roberts, \& Ream, E. (2013). A time to weep and a time to laugh: Humour in the nurse-patient relationship in an adult cancer setting. Support Care Center, 22, 1295-1301. 


\section{ANNEXE A}

Évaluation dermatologique - À compléter par l'infirmière

Zone affectée par l'érythème ou l'irritation.

\begin{tabular}{|l|l|l|l|l|}
\hline Évaluation physique & Aucun & Léger & Modéré & Intense \\
\hline Érythème & & & & \\
\hline Douleur (peau ou muscle) & & & & \\
\hline Démangeaison & & & & \\
\hline
\end{tabular}

Zone affectée par l'érythème ou l'irritation.

\begin{tabular}{|l|l|l|l|l|}
\hline Évaluation physique & Aucune & Léger & Modéré & Intense \\
\hline Érythème & & & & \\
\hline Douleur (peau ou muscle) & & & & \\
\hline Démangeaison & & & & \\
\hline
\end{tabular}

Zone affectée par l'érythème ou l'irritation.

\begin{tabular}{|l|l|l|l|l|}
\hline Évaluation physique & Aucun & Léger & Modéré & Intense \\
\hline Érythème & & & & \\
\hline Douleur (peau ou muscle) & & & & \\
\hline Démangeaison & & & & \\
\hline
\end{tabular}

1. Utilisez-vous le pansement InterDry Ag actuellement? (Si non, passez aux questions 10 et 11).

2. Trouvez-vous le pansement simple à utiliser?

3. Le pansement soulage-t-il votre inconfort?

4. Avez-vous changé le pansement plus souvent qu'aux cinq jours?

5. Si vous ressentez de la douleur, le pansement l'a-t-il soulagée?

6. Le pansement est-il confortable?

7. Croyez-vous que le pansement a aidé la peau à guérir?

8. Vous sentez-vous mieux lorsque vous portez le pansement?

9. Pourquoi avez-vous cessé d'utiliser le pansement? (Ne s’applique que si la personne n'utilise pas le pansement.)

10. Utilisez-vous d'autres pansements ou hydratants?

11. Avez-vous des questions ou des préoccupations dont vous aimeriez discuter? 


\section{ANNEXE B}

Entrevue téléphonique (menée par l'infirmière)

Évaluation de suivi - InterDry Ag

Bonjour, pourrais-je parler à___ ?

Bonjour, mon nom est et j'appelle du BCCA pour faire le suivi de la première semaine d'utilisation du pansement InterDry Ag. Avez-vous quelques minutes pour discuter de l'évolution de votre état au cours des deux dernières semaines?

Tout d'abord, comment allezvous?

1. Utilisezvous le pansement InterDry Ag actuellement? (Si non, passez à la question 10.)

2. Trouvez-vous le pansement simple à utiliser?

3. La rougeur de votre peau s'est-elle améliorée ou détériorée depuis votre dernier traitement?

4. Depuis le dernier traitement, avez-vous des plaies ouvertes, ou autrement dit, des plaques suintantes?

5. Avez-vous changé le pansement plus souvent qu’aux cinq jours?

6. Ressentez-vous de la douleur? Si oui, diriez-vous que la douleur provient de la peau ou du muscle?

7. Le pansement est-il confortable?

8. Selon vous, le pansement a-t-il aidé la peau à guérir?

9. Le pansement soulage-t-il votre peau?

10. Pourquoi avez-vous cessé d'utiliser le pansement? (Ne s'applique que si la personne n'utilise pas le pansement.)

11. Utilisez-vous d'autres pansements ou crèmes?

Avez-vous d'autres questions ou préoccupations dont vous aimeriez discuter?

Merci de votre temps et de vos précieux commentaires. Nous allons nous revoir dans une semaine, à bonne journée. Au revoir.

. Si vous avez des questions ou des inquiétudes, téléphonez à une infirmière au 604-930-4053. Passez une 


\section{ANNEXE C}

Évaluation du pansement InterDry Ag par les patientes

\begin{tabular}{|c|c|c|c|c|c|c|}
\hline $\begin{array}{l}\text { Dans quelle mesure êtes- } \\
\text { vous d'accord avec l'énoncé? }\end{array}$ & $\begin{array}{l}\text { Fortement en } \\
\text { accord }\end{array}$ & En accord & Indécises & En désaccord & $\begin{array}{l}\text { Fortement en } \\
\text { désaccord }\end{array}$ & $\begin{array}{l}\text { Ne s'applique } \\
\text { pas }\end{array}$ \\
\hline \multicolumn{7}{|l|}{$\begin{array}{l}\text { 1. InterDry Ag est simple à } \\
\text { utiliser. }\end{array}$} \\
\hline \multicolumn{7}{|l|}{$\begin{array}{l}\text { 2. InterDry Ag soulage la } \\
\text { douleur. }\end{array}$} \\
\hline \multicolumn{7}{|l|}{$\begin{array}{l}\text { 3. InterDry Ag soulage la } \\
\text { démangeaison. }\end{array}$} \\
\hline \multicolumn{7}{|l|}{$\begin{array}{l}\text { 4. Le pansement est } \\
\text { confortable. }\end{array}$} \\
\hline \multicolumn{7}{|l|}{$\begin{array}{l}\text { 5. Le pansement soulage } \\
\text { ma peau. }\end{array}$} \\
\hline \multicolumn{7}{|l|}{$\begin{array}{l}\text { 7. Le pansement a soulagé la } \\
\text { sensation de brûlure. }\end{array}$} \\
\hline \multicolumn{7}{|l|}{$\begin{array}{l}\text { 8. J'ai gardé le pansement } \\
\text { pendant } 5 \text { jours sans avoir à } \\
\text { le remplacer. }\end{array}$} \\
\hline \multicolumn{7}{|l|}{$\begin{array}{l}\text { 9. Je suis plus confortable } \\
\text { lorsque je porte le } \\
\text { pansement. }\end{array}$} \\
\hline
\end{tabular}

11. Avez-vous d'autres commentaires sur l'utilisation du pansement InterDry Ag? 